Service social

\title{
Domicile ou hébergement? Quand les croyances prennent le dessus
}

\section{Sylvie Rhéault}

Volume 43, numéro 1, 1994

Maintien à domicile

URI : https://id.erudit.org/iderudit/706641ar

DOI : https://doi.org/10.7202/706641ar

Aller au sommaire du numéro

Éditeur(s)

École de service social de l'Université Laval

ISSN

1708-1734 (numérique)

Découvrir la revue

Citer cet article

Rhéault, S. (1994). Domicile ou hébergement? Quand les croyances prennent le dessus. Service social, 43(1), 33-46. https://doi.org/10.7202/706641ar

\section{Résumé de l'article}

Les évaluations empiriques de l'efficience du maintien à domicile et de l'hébergement institutionnel tirent des conclusions souvent contradictoires ou vont à l'encontre des discours communément véhiculés. Cet article illustre, à partir de quelques exemples, les résultats des évaluations à la lumière des méthodes évaluatives utilisées, des variables de coûts incluses ainsi que des objectifs retenus par les équipes de recherche. 


\title{
Domicile ou hébergement? Quand les croyances prennent le dessus
}

\author{
Sylvie RHEAULT \\ Économiste \\ Direction de l'évaluation \\ Ministère de la Santé et des Services sociaux
}

\section{INTRODUCTION}

L'évaluation économique demeure difficile à introduire dans le domaine socio-sanitaire malgré les nombreux discours sur l'amélioration de I'efficience. L'une des raisons est que les évaluations économiques rendent les choix d'allocation de ressource plus explicites, que ces choix $s^{\prime}$ effectuent dans un contexte de rationnement budgétaire ou non.

Depuis de nombreuses années, on soutient que le maintien à domicile, surtout celui des personnes âgées en perte d'autonomie, est la solution la plus efficiente. Dans ce contexte, introduire une information qui relativise cette donnée peut être mal perçu par ceux et celles qui croient fermement en ce mode d'intervention pour améliorer la santé et la qualité de vie des personnes âgées.

* L'auteure tient à remercier ses collègues Lysette Trahan-Langlois, Gilles-Yvon Picard et Alain Saucier de la Direction générale de la planification et de l'évaluation du ministère de la Santé et des Services sociaux pour leurs précieux commentaires sur la version préliminaire de cet article. 
D'abord, précisons certains faits. Les évaluations économiques doivent, dans la mesure du possible, tenir compte à la fois des coûts et des résultats en matière de santé et de bien-être pour conclure à l'efficience d'un mode d'hébergement des personnes âgées en perte d'autonomie. Ainsi, des coûts plus élevés n'impliquent pas nécessairement une moins grande efficience. Des résultats additionnels plus élevés que ces coûts supplémentaires permettent de conclure, dans ce cas, à une plus grande efficience. De plus, les évaluations économiques n'intègrent pas l'ensemble des éléments à considérer à des fins de décision. Rien n'interdit, et c'est souvent le cas dans notre système sociosanitaire, que des décisions s'appuient sur des considérations éthiques ou d'équité au détriment de l'efficience. Cette possibilité s'avère d'ailleurs très rassurante pour la population âgée.

Par ailleurs, les évaluations économiques disponibles sur les modes d'hébergement des personnes âgées en perte d'autonomie ne tranchent pas définitivement en faveur du maintien à domicile ou de I'hébergement institutionnel. Tout au plus précisent-elles que, pour certaines clientèles, un mode d'intervention peut être préférable à l'autre ou bien que les choix d'allocation de ressources gouvernementales et privées dépendent des objectifs politiques poursuivis par les programmes. Enfin, ces évaluations nous rappellent que la comparaison des dépenses publiques effectivement effectuées à domicile ou en institution ne constituent pas une information valable pour une prise de décision. En effet, se servir de cette information ne fait que confirmer les décisions déjà prises en matière d'allocation des ressources. Il faut plutôt faire référence aux coûts réels et non aux seules dépenses déjà engagées.

\section{QUE PEUVENT NOUS DIRE LES ÉVALUATIONS ÉCONOMIQUES RÉALISÉES JUSQU'ICI?}

La réponse dépend d'abord et avant tout de l'objectif poursuivi par les évaluations économiques, de la méthode et de la rigueur de ces évaluations.

Ainsi, lorsque l'objectif de l'évaluation est de voir en quoi le maintien à domicile permet d'éviter des dépenses publiques plus importantes en matière $d^{\prime}$ institutionnalisation et $d$ 'hospitalisation, l'évaluation se limite aux coûts publics. Par contre, lorsqu'on veut allouer les ressources de la collectivité de façon optimale, l'évaluation tiendra compte autant des coûts que des avantages, qu'ils soient privés ou publics.

L'efficience sera donc mesurée de façon différente selon I'objectif poursuivi. Généralement, les économistes préfèrent une évaluation qui 
a une perspective sociale plutôt qu'uniquement gouvernementale. Une telle évaluation permet, en effet, d'orienter I'allocation des ressources limitées, tant publiques que privées, de façon optimale et de discuter également du partage du fardeau du financement des services.

Divers aspects méthodologiques doivent être pris en compte pour la comparabilité des coûts et des résultats. Dans un premier temps, il est nécessaire de comparer des populations homogènes. Le niveau de dépendance et les caractéristiques socio-économiques des bénéficiaires sont les principales variables à considérer.

Tous les coûts, tant privés que publics, financiers que non financiers, doivent, dans la mesure du possible, être inclus. Par exemple, les dépenses privées en alimentation et en hébergement doivent être considérées autant pour les personnes vivant à domicile que pour celles en institution.

L'évaluation doit comprendre certaines activités non rémunérées, tels le temps de travail domestique et le bénévolat. En effet, une analyse qu'on veut économique ne peut, en soi, exclure de son champ ces activités. L'existence d'une rémunération ne donne pas à un bien ou à un service une valeur économique ; c'est plutôt l'existence des échanges de biens et de services dans la population, dans un contexte de rationnement, qui constitue cette valeur.

Or, la présence du travail domestique et du bénévolat explique probablement une bonne part de la variance des coûts entre les modes $d^{\prime}$ hébergement institutionnel et le maintien à domicile. Ainsi, exclure de l'analyse des coûts les composantes bénévoles et domestiques réduit nos possibilités d'explication pour les écarts de coûts financiers entre les divers modes d'hébergement. De plus, exclure cette dimension nous empêche de connaître le partage du fardeau du financement entre le gouvernement et le secteur privé, selon les modes d'hébergement.

Également, dans le milieu institutionnel, une attention spéciale doit être prêtée aux coûts fixes (immobilisations), aux coûts communs d'un département à l'autre et aux coûts variables correspondant à I'intensité de services selon la dépendance de la personne âgée.

Relativement aux résultats, il importe de clarifier les objectifs poursuivis par les évaluations pour bien les mesurer. Cette clarification permet également de mieux choisir la méthode d'évaluation de l'efficience.

Dans le cas où plusieurs objectifs sont poursuivis et où il est possible de les comptabiliser en dollars, les analyses coûts-avantages peuvent être retenues. Les avantages directs sont alors mesurés par les coûts évités provenant de la réduction des coûts du système sociosanitaire à la suite de l'implantation ou du fonctionnement d'un service 
ou programme. Les avantages indirects sont constitués des gains de productivité, de la valeur du temps de loisir additionnel... Le plus souvent, les auteurs se restreignent à la dimension des avantages directs. C'est I'approche retenue dans la plupart des études américaines sur l'évaluation économique des programmes de maintien à domicile.

Pour des objectifs particuliers mesurables uniquement en unités naturelles comme l'espérance de vie ou les durées de séjour en milieu hospitalier, I'analyse coûts-efficacité convient davantage. Finalement, lorsqu'il s'agit de mesurer des préférences ou d'intégrer des variables de qualité de vie à la variable espérance de vie, les analyses coûts-utilité sont à retenir. Toutefois, dans le domaine de I'hébergement pour les personnes âgées en perte $d^{\prime}$ autonomie, cette dernière méthode d'évaluation n'a pas, à notre connaissance, été utilisée jusqu'ici.

II n'est pas toujours possible ni nécessaire d'effectuer une évaluation qui porte à la fois sur les résultats et sur les coûts. Par exemple, lorsque nous savons que les résultats recherchés et finalement obtenus par les divers modes d'hébergement sont équivalents, une approche de minimisation des coûts s'avère suffisante. Dans ces cas cependant, la comparaison de services équivalents devient une condition sine qua non à une comparaison valide des coûts. On ne peut, en effet, conclure à des coûts moindres alors que ces coûts sont plus bas uniquement en raison de services moindres.

Ces éléments méthodologiques doivent être soulignés à l'occasion $d^{\prime}$ une recension des évaluations économiques parce qu'ils permettent de porter un jugement sur la valeur des données ainsi que sur la comparabilité des résultats des évaluations. Ils éclairent aussi quant à portée des évaluations aux fins décisionnelles.

Dans la section qui suit, nous illustrons l'impact possible de ces évaluations économiques sur les décisions gouvernementales. Ces illustrations couvrent trois méthodes d'évaluation : analyse coûtsavantages, analyse coûts-efficacité et minimisation des coûts.

\section{QUELQUES EXEMPLES D'APPLICATION}

\section{Analyse coûts-avantages}

Pour mesurer le rapport coûts-avantages des programmes de maintien à domicile, on compare généralement deux groupes vivant à domicile, l'un bénéficiant des programmes traditionnels de maintien à domicile et l'autre d'un programme bonifié. La principale bonification consiste en la coordination de l'ensemble des services. Les avantages considérés sont liés à la réduction de l'institutionnalisation et de 
I'hospitalisation des personnes âgées en perte d'autonomie. On compare essentiellement les coûts des programmes publics.

Les avantages sont ainsi calculés par les coûts évités. Pour connaître les avantages nets du programme, on soustrait des coûts évités les coûts additionnels amenés par ce programme.

Dans ces évaluations, le maintien à domicile des personnes en perte d'autonomie est considéré a priori comme moins dispendieux que le maintien en hébergement institutionnel. Cette hypothèse, même si elle se révèle fondée, $n^{\prime}$ invalide cependant pas la question du coût moindre, ou non, du maintien à domicile par rapport à l'institutionnalisation. En effet, selon cette approche, pour conclure à l'efficience des programmes de maintien à domicile il faut que les coûts évités soient collectivement plus élevés que les coûts des nouveaux services de maintien à domicile. Pour atteindre cet objectif, il ne faut pas que les programmes de maintien à domicile s'adressent à une nouvelle clientèle; ces programmes doivent viser les personnes qui autrement seraient hospitalisées ou institutionnalisées.

Weissert et Cready (1989) ont regroupé les résultats de 31 recherches effectuées aux États-Unis, depuis le début des années 60. Leur recension a été exécutée à partir de 700 titres et recherches sur le sujet. Les auteurs ont retenu uniquement les études répondant aux conditions suivantes:

- évaluation de services bonifiés de maintien à domicile par rapport aux services traditionnels ; le plus souvent, les services traditionnels sont ceux des programmes de base de maintien à domicile offerts par Medicaid ;

- utilisation d'une structure expérimentale, soit un groupe témoin et un groupe bénéficiant du programme ;

- un nombre minimum de 50 personnes par groupe ;

- I'individu vu comme l'unité d'analyse;

- programmes visant principalement les personnes âgées.

Les données regroupées portent sur un an et la période de référence est janvier 1988. Tant les services offerts que les coûts considérés dans les diverses études diffèrent. Le plus souvent, il s'agit uniquement des coûts publics. Aucune correction n'est apportée pour tenir compte de ces différences.

On obtient les augmentations ou diminutions dans I'utilisation des services (centres d'hébergement, centres hospitaliers, services ambulatoires), ainsi que les coûts. On a aussi calculé les coûts additionnels entraînés par la mise en place d'un programme à domicile. 
Les résultats nets proviennent de la différence, dans l'utilisation des divers services, entre les groupes témoins et les groupes expérimentaux.

Les deux auteurs ont comparé les résultats des études menées avant 1980 avec celles faites après 1980. Les expériences postérieures à 1980 ont mieux réussi à cibler la clientèle à haut risque d'hébergement, donc peuvent mieux permettre de réduire les coûts publics. Pourtant, les coûts nets sont plus élevés : 2357 \$ comparativement à $962 \$$ additionnels.

\section{TABLEAU 1}

Les résultats sommaires des évaluations américaines des trente dernières années (moyenne pondérée)*

\begin{tabular}{lcc}
\hline & \multicolumn{2}{c}{ Coût per capita } \\
& Groupe témoin & Groupe expérimental \\
\hline Centre d'hébergement & $3067 \$$ & $1662 \$$ \\
Hospitalisation & $5163 \$$ & $5237 \$$ \\
Soins ambulatoires & $1985 \$$ & $2024 \$$ \\
Programme à domicile & $1697 \$$ & $5147 \$$ \\
Coût net comparé & & $+1709 \$$ \\
& & \\
* Les différences ont été calculées en utilisant également la moyenne non pondérée et la \\
médiane ; les résultats ne diffèrent pas significativement.
\end{tabular}

Source: Weissert et Cready, op. cit., 1989.

Donc, dans la plupart des études menées au cours des trente dernières années aux États-Unis, les programmes de maintien à domicile n'ont pas eu pour effet de réduire les coûts. En effet, les coûts additionnels attribuables aux programmes ont généralement dépassé les gains obtenus par la réduction dans l'utilisation des services institutionnels.

Par ailleurs, dans les trente et une études examinées, on trouve diverses mesures de résultats: taux de survie, capacité à exercer les activités quotidiennes, facultés cognitives, capacité mentale, comportement psychosocial, satisfaction dans la vie, participation à des activités sociales, interaction sociale, effet sur les aidantes ou aidants naturels et conséquences sur les besoins non comblés.

Le taux de survie et le niveau de capacité mentale ont été, dans certains cas, améliorés, quoique faiblement, par l'existence de services communautaires. Les résultats ne sont cependant pas concluants. Les effets sur les capacités physiques ont beaucoup été mesurés, mais aucune conclusion globale $\mathrm{n}^{\prime}$ en ressort. 
L'augmentation de la satisfaction dans la vie semble constante dans les études chez les personnes qui ont bénéficié du programme. Les aidantes ou les aidants naturels semblent aussi avoir apprécié grandement ces programmes. Globalement, on observe que les personnes bénéficiant du programme ont moins de besoins non comblés et s'intègrent mieux socialement. Mais, là encore, ces effets sont faibles et les conditions qui rendent les évaluations solides sont absentes.

Quelle solution devrait-on privilégier à la lecture de ces informations? Serait-il approprié de mettre en œuvre et de bonifier les programmes de maintien à domicile? La réponse «administrative » penche naturellement vers la négative.

Or, rappelons que ces évaluations visent à connaître l'impact des programmes «publics » de maintien à domicile sur l'ensemble des coûts publics. On recherche d'abord et avant tout une substitution de services, soit un maintien à domicile au lieu d'un séjour institutionnel. On ne vise nullement les clientèles à faible risque d'institutionnalisation. Pourtant, l'efficience comparée du maintien à domicile et de l'institutionnalisation sert non seulement à favoriser des substitutions, mais également à améliorer l'allocation des nouvelles ressources gouvernementales.

Par ailleurs, ces études se sont limitées à une perspective gouvernementale. Les dépenses privées ainsi que les efforts déployés par les proches auprès des personnes âgées ne sont pas pris en compte. Or, il est possible, du point de vue de l'allocation de l'ensemble des ressources, tant publiques que privées, qu'un développement accru des programmes de maintien à domicile soit approprié, du moins pour certaines catégories de bénéficiaires.

L'autre élément qui doit être mis en exergue est que l'évaluation de la qualité des services et de la satisfaction des bénéficiaires mérite des efforts additionnels sur le plan de la recherche évaluative. Rappelons que les études coûts-avantages devraient inclure non seulement les avantages directs, tels les coûts évités en institutionnalisation, mais aussi les avantages indirects tels que l'amélioration des conditions de vie et de revenu des proches.

Bref, ces évaluations nous éclairent sur les gains potentiels de ce programme sur les coûts publics à court terme, ce qui constitue bien entendu une information très utile pour les décideurs. Mais elles demeurent partielles du point de vue d'une allocation efficiente des ressources.

\section{Analyse coûts-efficacité}

Alors que les études américaines ont généralement évalué le rapport coûts-avantages d'un programme bonifié de maintien à domicile, 
relativement aux services traditionnels pour les personnes âgées vivant à domicile, une étude britannique (Challis et al., 1991) a évalué le rapport coûts-efficacité d'un programme bonifié de maintien à domicile relativement aux services institutionnels de longue durée.

On a comparé deux groupes de personnes sortant d'un séjour hospitalier de courte durée, l'un se trouvant dans un établissement de longue durée et l'autre bénéficiant du programme bonifié de maintien à domicile. La bonification des services traditionnels de maintien à domicile portait principalement sur deux aspects: coordination des services par une équipe gériatrique, soutien additionnel pour les aidants naturels. Le suivi a été effectué sur une période de douze mois.

Certaines différences ont été constatées entre les deux groupes. Après avoir corrigé les données, on n'a observé aucune différence entre les groupes, en ce qui a trait aux décès. Par contre, après six mois, les dépressions étaient moins nombreuses parmi le groupe à domicile et les indices de qualité de vie étaient généralement meilleurs. Par ailleurs, on n'a pas constaté d'augmentation de stress chez les aidants naturels lorsque les personnes âgées étaient à domicile.

Tant les dépenses publiques que les dépenses privées ont été considérées. Les coûts d'immobilisations ont aussi été inclus. Par contre, si les dépenses privées des aidants ont été considérées, il n'en est pas de même pour la valeur du travail bénévole effectué auprès des siens. Au total, les dépenses hebdomadaires étaient de $277 £$ à $424 £$ pour le groupe institutionnalisé et de $242 £$ à $254 £$ pour le groupe expérimental, soit vivant à domicile.

Les auteurs ont conclu à une plus grande efficacité et à de plus faibles coûts du programme bonifié de maintien à domicile comparativement aux services traditionnels.

Si cette conclusion peut réconforter les tenants du maintien à domicile, elle $n^{\prime}$ est toutefois pas sans risque pour le développement du maintien à domicile, si on l'applique sans nuance. En effet, cette étude ne tient pas compte des différences possibles entre les groupes relativement à la perte d'autonomie. Or, tant les résultats liés aux coûts que ceux associés aux avantages pourraient être modifiés. Par exemple, la charge de travail pour les proches est beaucoup plus lourde pour les personnes en grande perte d'autonomie. Une standardisation des données en fonction de la perte d'autonomie pourrait conduire à une plus grande augmentation du stress chez les aidants, à moyen et à long terme, pour cette catégorie de bénéficiaires.

De plus, le fait de ne pas considérer le travail bénévole implique l'hypothèse que cette ressource est illimitée et toujours disponible. Or, un développement accru du maintien à domicile pourrait entraîner 
des pénuries. Pour une allocation efficiente des ressources, il est donc préférable de tenir compte de la valeur de cette activité, même si la majorité de la population reconnaît que ces activités ne doivent pas être rémunérées.

\section{Minimisation des coûts}

Selon cette méthode, les populations comparées vivent dans des lieux d'hébergement différents. L'intérêt de ces études porte davantage sur la comparaison des coûts des divers modes d'hébergement existants que sur les avantages ou désavantages que procure un mode d'hébergement relativement à une situation de référence. Ces études se limitent aussi à la dimension coût; le volet « résultat» des divers modes d'hébergement n'est pas considéré.

Aux fins d'illustration, deux études seulement sont retenues. La première (Sager, 1983) évalue des coûts hypothétiques de maintien à domicile pour une population hospitalisée, l'autre (Bouget et al., 1990) compare plusieurs modes d'hébergement et remplit bon nombre des conditions énoncées pour une comparaison complète des coûts.

Sager (1983) a évalué les coûts d'un maintien fictif à domicile pour un groupe de bénéficiaires d'un hôpital de soins aigus orientés à la sortie vers divers centres d'hébergement pour personnes âgées. Les coûts de cette situation hypothétique sont alors comparés aux coûts réels en centre d'hébergement.

Les critères retenus pour la comparaison de populations équivalentes ne pourraient être mieux remplis. En effet, le chercheur a choisi une clientèle hébergée pour laquelle un coût fictif du maintien à domicile est évalué. Ainsi, que ce soit pour les coûts à domicile ou en hébergement, il s'agit des mêmes personnes. De plus, en établissant une grille fictive de services les plus complets possible, pour permettre à une personne de demeurer à domicile, les services à domicile et en hébergement institutionnel sont davantage comparables.

Les coûts du maintien à domicile sont établis à partir du nombre de services considérés comme nécessaires par les professionnels et professionnelles de la santé. Des taux horaires sont, par la suite, imputés selon les compétences exigées par ce travail. Cette grille de services est remplie pour chaque personne âgée, à partir d'une évaluation de son état de santé et de ses capacités fonctionnelles et psychosociales. Dans le total des coûts, le nombre d'heures de services qui peuvent être offerts de façon bénévole est exclu. Par ailleurs, on impute un certain montant pour le logement. 
Que l'on considère les coûts médians ou moyens, le maintien à domicile est plus dispendieux. Les coûts moyens institutionnels s'élèvent entre 373 \$ et 410 \$ par semaine, selon qu'il s'agit des coûts excluant ou non les jours passés en centre hospitalier, en attente d'un hébergement. Les coûts médians à domicile s'élèvent à 513 \$ et les coûts moyens à $574 \$$ par semaine.

Les vérifications faites par l'auteur portent à croire qu'il s'agit d'une population qui souffre d'une grande perte d'autonomie. Ainsi, cette conclusion ne pourrait être transposée à l'ensemble des personnes âgées. Par ailleurs, en excluant le travail bénévole, Sager se trouve à sous-estimer, relativement aux coûts institutionnels, les coûts du maintien à domicile. L'auteur reconnaît toutefois que les heures prescrites théoriquement par le personnel professionnel semblaient élevées, surestimant possiblement les coûts du maintien à domicile. L'effet net est donc incertain.

Bouget et al. (1990) ont cherché à comparer des populations

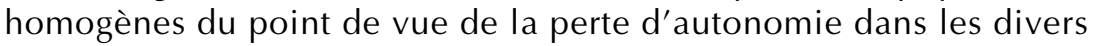
milieux de vie des personnes âgées (domicile privé, logement-foyer, maison de retraite, avec ou sans cure médicale, et long séjour en institution). Ils ont tenu compte des variables socio-économiques pour la comparaison des populations.

Les dépenses de consommation courante, tant privées que publiques, sont comprises. Certaines dépenses non observables directement ont été imputées: les exonérations de paiement pour les personnes âgées et les logements fournis gratuitement par un membre de la famille. Une valeur a également été attribuée au travail domestique et au bénévolat.

Les coûts institutionnels ont été ajustés pour tenir compte du temps accordé aux bénéficiaires sous forme d'aide et de soins divers selon le degré de dépendance des personnes âgées. Seules les dépenses variant en fonction de la dépendance ont été considérées. On a ainsi pu comparer les coûts des divers modes d'hébergement par la mesure du niveau de dépendance.

Ainsi, les coûts de cinq modes d'hébergement ont été comparés dans l'étude de Bouget et al. On y conclut globalement que le maintien à domicile privé est moins dispendieux : il coûte, en moyenne, 5714 francs par mois par bénéficiaire (octobre 1988). Le logementfoyer coûte 6150 francs ; la maison de retraite sans cure médicale, 6883 francs; la maison de retraite avec cure médicale, 7351 francs et, finalement, le long séjour coûte 11324 francs par mois. On examine également le lien entre les coûts et les niveaux de dépendance.

Les deux tableaux suivants résument les résultats. Dans le tableau $2 \mathrm{~A}$, on a restreint les coûts à l'aide et aux soins de santé, alors que dans 
Tableau 2A

Comparaison des dépenses globales d'aide et de santé Dépenses moyennes par bénéficiaire (francs par mois)*

\begin{tabular}{lccccc}
\hline Dépendance & $\begin{array}{c}\text { Domicile } \\
\text { privé }\end{array}$ & $\begin{array}{c}\text { Logement } \\
\text { Foyer }\end{array}$ & $\begin{array}{c}\text { MR } \\
\text { sans SCM }\end{array}$ & $\begin{array}{c}\text { MR } \\
\text { avec SCM*** }\end{array}$ & $\begin{array}{c}\text { Long } \\
\text { séjour }\end{array}$ \\
\hline Nulle & 1680 & 1484 & 2120 & 1392 & \\
$\begin{array}{l}\text { Très faible } \\
\text { Faible }\end{array}$ & 2186 & & 1433 & 1960 & \\
Moyenne & 2672 & 3032 & 1732 & 1970 & 3105 \\
$\begin{array}{l}\text { Forte } \\
\text { Très forte }\end{array}$ & 3535 & 3821 & 2139 & 2353 & 5829 \\
Extrême & 5167 & 2349 & 2798 & 5801 \\
\hline
\end{tabular}

Tableau 2B

Comparaison des dépenses totales

Dépenses moyennes par bénéficiaire (francs par mois)*

\begin{tabular}{|c|c|c|c|c|c|}
\hline Dépendance & $\begin{array}{c}\text { Domicile } \\
\text { privé }\end{array}$ & $\begin{array}{l}\text { Logement } \\
\text { Foyer }\end{array}$ & $\begin{array}{c}\mathrm{MR} \\
\text { sans } \mathrm{SCM}^{* *}\end{array}$ & $\begin{array}{c}\mathrm{MR} \\
\text { avec SCM } \mathrm{SM}^{* * *}\end{array}$ & $\begin{array}{l}\text { Long } \\
\text { séjour }\end{array}$ \\
\hline Nulle & 4953 & \multirow{2}{*}{5340} & \multirow{2}{*}{6810} & \multirow{2}{*}{6220} & \multirow{5}{*}{8322} \\
\hline Très faible & 5179 & & & & \\
\hline Faible & 5587 & \multirow{2}{*}{6857} & 5764 & 6795 & \\
\hline Moyenne & 5892 & & 6268 & 6708 & \\
\hline Forte & 6336 & \multirow{3}{*}{7882} & 6489 & 7058 & \\
\hline Très forte & 7462 & & 6464 & 7589 & 11192 \\
\hline Extrême & 9063 & & 8498 & 8617 & 11851 \\
\hline
\end{tabular}

* Pour les bénéficiaires seulement ; soit $70 \%$ de ceux à domicile, 86 \% en logement-foyer et $100 \%$ en institution.

** Maison de retraite sans cure médicale.

*** Maison de retraite avec cure médicale.

Source : Bouget et al., op. cit., 1990.

le tableau 2B toutes les dépenses sont incluses. Nous pouvons voir que, pour les niveaux élevés de dépendance, le domicile se compare facilement avec le long séjour, lorsque seules les dépenses liées aux soins et services sont considérées. Par ailleurs, les maisons de retraite possèdent un avantage comparatif pour les coûts liés à l'aide et aux soins, surtout pour les niveaux de dépendance très forte ou extrême. 
Pour les dépenses globales, le domicile privé apparaît moins dispendieux que le long séjour. Toutefois, il ne coûte pas systématiquement moins cher, en moyenne, que l'hébergement en logementfoyer ou en maison de retraite. Le long séjour demeure toujours le mode d'hébergement le plus onéreux.

Cette étude n'a pas abordé la question de l'équivalence des services fournis. Ainsi, rien ne nous assure que les différences de coûts ne sont pas attribuables à une quantité moindre ou supérieure de services. La prise en considération du travail domestique et du bénévolat permet de réduire ces différences, mais sûrement pas de les annuler. Par ailleurs, cette évaluation ne tient pas compte des différences de résultats à l'égard de la santé et du bien-être.

\section{QUE POUVONS-NOUS TIRER DE CET EXERCICE POUR LE QUÉBEC?}

À la lecture des diverses évaluations et tenant compte des différences méthodologiques importantes entre celles-ci, il apparaît difficile de conclure définitivement, du point de vue de l'efficience, en faveur d'un mode d'hébergement. De plus, considérant les différences structurelles d'un pays à l'autre et relativement au Québec, il s'avère imprudent d'utiliser ces informations à des fins d'allocation des ressources au Québec. Toutefois, ces évaluations demeurent très éclairantes sur le plan des aspects méthodologiques et elles soulèvent des pistes fort intéressantes.

Pour mieux comprendre la situation québécoise, une évaluation des besoins, des ressources requises et des coûts des divers milieux de vie des personnes âgées est actuellement en cours au Québec (Saucier et Rheault, 1992 ; Hébert et al., 1993). Pour réaliser l'objectif d'allouer les ressources de façon optimale, il devenait nécessaire de comparer tous les milieux de vie des personnes âgées, en passant du maintien à domicile sans services gouvernementaux au lit de longue durée dans les centres hospitaliers psychiatriques. De plus, considérant que le partage du financement, entre le secteur public et les particuliers, comporte actuellement certains choix politiques, il était nécessaire d'inclure l'ensemble des coûts (dépenses publiques, dépenses privées et bénévolat), pour ne pas biaiser les comparaisons. Toutefois, pour faciliter les discussions sur le partage du fardeau du financement, l'évaluation distingue, pour l'analyse des ressources fournies, le partage des coûts entre le secteur public, les bénéficiaires et le secteur bénévole.

Les besoins, les ressources requises et fournies, de même que les coûts, seront comparés d'un milieu de vie à l'autre, pour des popu- 
lations semblables du point de vue de I'autonomie. Ainsi, il sera possible de connaître les coûts des divers milieux de vie pour des populations équivalentes. Par ailleurs, seront comparés les coûts pour des services équivalents. En effet, à partir des niveaux de dépendance, une évaluation des ressources requises et des coûts qui y sont associés est réalisée. Le lien entre des populations équivalentes, des services équivalents et les coûts, selon les divers milieux de vie, sera donc possible.

L'évaluation québécoise met de côté les résultats. On suppose a priori que des services équivalents, pour des populations équivalentes, donneront des résultats équivalents. Une étude ultérieure serait donc nécessaire pour valider cette hypothèse. $\mathrm{D}^{\prime} \mathrm{ici}$ là, nous aurons cependant une information qui pourra nous donner l'heure juste sur les coûts comparés des modes d'hébergement.

Les résultats de l'évaluation québécoise devraient être disponibles au printemps de 1996.

\section{Références bibliographiques}

Bouget, Denis, Robert TARTARIN, Michel Frossard et Pierre TRIPIER (1990). Le prix de la dépendance. Comparaison des dépenses des personnes âgées selon leur mode d'hébergement. Paris, Caisse nationale d'assurance-vieillesse des travailleurs salariés, $414 \mathrm{p}$.

CHALLIS, D. et al. (1991). "An Evaluation of an Alternative to Long-Stay Hospital Care for Frail Elderly Patients: "Costs and Effectiveness" Age and Agency», $20: 245-254$.

FROSSARD, Michel et Bernard ENNUYER (1987). Comparaison des coûts de I'hébergement médico-social et du maintien à domicile des personnes âgées. Paris, Éditions École nationale de la santé publique, 76 p., Collection Études et Recherches.

HÉBERT, Réjean et al. (1993). Évaluation de certaines ressources requises pour les personnes âgées en perte d'autonomie. Centre de recherche en gérontologie et gériatrie. Sherbrooke, février (document interne).

RHEAULT, Sylvie (1994). L'évaluation économique des modes d'hébergement pour les personnes âgées en perte d'autonomie : Recension des méthodes et des résultats. Québec : Ministère de la Santé et des Services sociaux, Direction de la planification et de l'évaluation, $87 \mathrm{p}$.

SAGER, A. Peter (1983). Planning Home Care with the Elderly. Patient, Family and Professional Views of an Alternative to Institutionalization. Cambridge: Ballinger Publishing Company, 295 p.

SAUCIER, Alain et Sylvie RHEAULT (1992). Évaluation de certaines ressources requises pour les personnes âgées en perte d'autonomie. Ministère de la Santé et des Services sociaux, Direction de l'évaluation, juillet, 25 p. 
Weissert, William G. et Cynthia Cready (1989). "A Prospective Budgeting Model for Home and Community-Based Long-Term Care», Inquiry, vol. $26: 116-129$.

Weissert, William G., Cynthia Cready et James E. PaWelaK (1988). "The Past and Future Home and Community-Based Long-Term Care », The Milbank Quarterly, vol. 66, n² 2 :309-388. 